\title{
Breakthrough in heart xenotransplantation
}

the procedure
had a high
consistency of
transplantation
survival,
with four of
five animals
in the final
experiment
surviving for
$\geq 3$ months

Baboons receiving heart replacement with genetically modified pig hearts have survived for up to 195 days after transplantation, according to a study published in Nature. This modified protocol for cross-species transplantation (xenotransplantation) developed by Bruno Reichart and colleagues is a great improvement on previous efforts - the longest survival so far of a pig-to-baboon heart replacement was only 57 days - and brings us a step closer to realizing the goal of pig-to-human heart transplantation. "This efficacy study opens widely the door to clinical applications," says Reichart.

Heart transplantation is the only option for patients with terminal heart failure. The outcomes with transplantation of human hearts are good and life is not only prolonged but quality of life also improves. However, there is a severe organ shortage worldwide, and the number of organs available does not meet the demand for heart transplantation. Therefore, alternative techniques are necessary. "Mechanical assist devices could be a solution but have serious adverse effects," explains Reichart. "By contrast, xenogeneic heart transplantations are possible and surprisingly easy to manage in the long-term, especially after using state-of-the-art, non-nephrotoxic immunosuppression," he remarks.

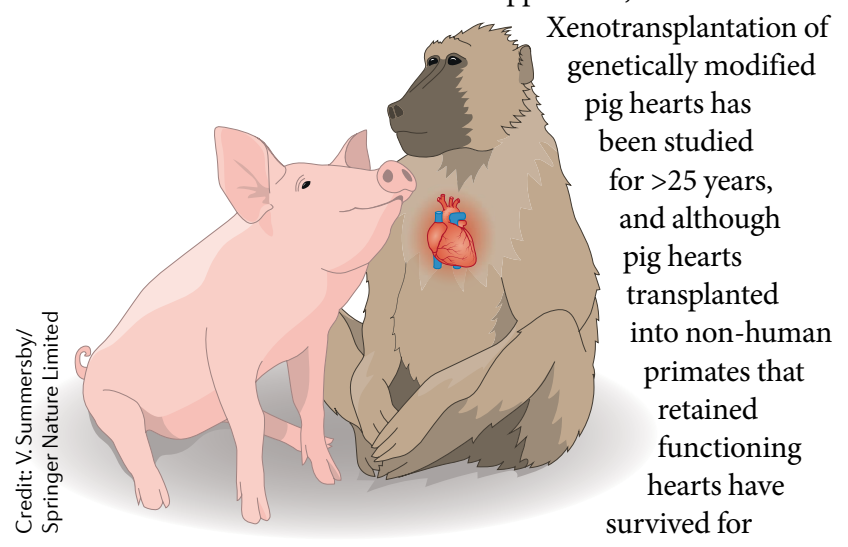

945 days, life-supporting pig hearts lasted only 57 days.

To increase the survival of pig-tobaboon transplantations, Reichart and colleagues used a previously described immunosuppression protocol and optimized the xenotransplantation procedure. First, the researchers used pig hearts that were genetically modified to reduce immune rejection. The engineered pig hearts lacked galactose- $\alpha$-1,3-galactose epitopes (which can induce immune reactions against organs transplanted from pigs into humans and other primates) and expressed the human proteins CD46 (which blocks the immune response) and thrombomodulin (which prevents blood clotting after surgery). In addition, the baboons were treated with a previously described immunosuppression treatment before receiving the transplantation.

The investigators highlight that two steps were crucial to ensure the long-term function of the xenograft in baboons. First, the engineered pig hearts require non-ischaemic preservation with continuous perfusion of an oxygenated solution of blood and nutrients, instead of cold static storage, which is the standard for human organ transplantations. Second, post-transplantation overgrowth needs to be restricted. In initial experiments, the recipient animals died within 40 days as a result of massive cardiac overgrowth. Therefore, to prevent the detrimental cardiac growth, the baboons received treatment to lower their blood pressure to match that of pigs and thereby avoid heart enlargement, as well as a drug called temsirolimus to block cell growth and proliferation. In addition, treatment with the anti-inflammatory steroid cortisone, which is used for immunosuppression but can induce cardiac overgrowth, was withdrawn early after surgery.

After these modifications to the protocol, the procedure had a high consistency of transplantation survival, with four of five animals in the final experiment surviving for $\geq 3$ months. One had to be euthanized after 51 days, two lived for $\geq 3$ months and were healthy, and two were allowed to survive for 6 months before being euthanized.

In an accompanying commentary article, Christoph Knosalla (German Heart Center Berlin, Germany), who was not involved in the study, remarks that the blood-perfusion protocol used by Reichart and colleagues might be beneficial for human-to-human transplantations to improve results in the clinic and might allow an increase in the pool of donor hearts by including organs that are currently considered suboptimal. Knosalla points out that given that the immunosuppression protocol used in this study was well tolerated by the baboons, with no major immunosuppression-related infections, this method might also be safe for use in humans.

The next step for the research team is to continue the optimization of the transplantation procedure to achieve the goal of six long-term survivors for $\geq 3$ months out of ten experiments. "We need a better co-stimulation blockade for future human applications and 'safe' donor animals that will not cause any deleterious infectious diseases in human recipients," adds Reichart. Irene Fernández-Ruiz

ORIGINAL ARTICLE Längin, M. et al. Consistent success in life-supporting porcine cardiac xenotransplantation. Nature 564, 430-433 (2018) 